\section{Fristenregelung: sollen/müssen wir Ärztinnen und Ärzte Stellung nehmen?}

Ursula Steiner-König, Vizepräsidentin der FMH

\section{Panta rhei - alles ist im Fluss}

Vor einem halben Jahrhundert mochte noch gelten, dass es in Elternhaus, Schule und Kirche eine gemeinsam gelehrte moralische Norm gab, wie eine religiöse oder philosophische Verankerung Verzweiflung verhindern könne. Heute hingegen erleben wir - mehr denn je - wie "alles im Fluss ist", wie einer multikulturellen und pluralistischen Gesellschaft jeder Konsens in moralischen Fragen abhanden gekommen ist. Gerade dieser Verlust an Leitplanken innerhalb unserer Gesellschaft ruft dringend nach ethischen Überlegungen und Richtlinien. Würden wir allerdings von Ethik allgemein erwarten, dass sie uns Leitsätze von der Evidenz mathematischer Formeln zur Verfügung stellen sollte, wären wir genauso auf dem Holzweg, wie wenn wir sie reduzieren würden auf schlicht unüberprüfbare Gefühle und Emotionen. Es braucht - so Bondolfi [1] - eine «mittlere Position, welche die These vertritt, dass ethische Diskurse eine gewisse Plausibilität aufweisen, welche zwischen puren Gesinnungen einerseits und nackten Tatsachen andererseits anzusiedeln ist».

Im nachfolgenden möchte ich - anhand von ausgewählten Bruchstücken ethischer Reflexionen einige Überlegungen zum Thema Schwangerschaft und Schwangerschaftsabbruch anstellen.

Wenigstens in unserem Kulturkreis haben Achtung der Persönlichkeit, des Willens und der Rechte des Einzelnen an Bedeutung gewonnen. Medizinethik als angewandte Ethik ist bezogen auf das ärztliche Handeln am kranken Menschen, ist "nicht einfach zweckrationales Handeln, sondern ein kommunikatives Handeln" [1], welches nur in der Arzt-PatientenBeziehung seine Entfaltung findet. Das Praktische ist immer impliziert. Damit wird medizinisches Wissen zu einem Resultat zwischen naturwissenschaftlichem Beobachten und ethisch motiviertem Intervenieren.

Gemäss des Eides des Hippokrates geht es beim medizinischen Handeln immer um das «primum nihil nocere». Kommt es zu einem ethischen Konflikt, sind heute - stärker als früher - medizinische und nichtmedizinische Bereiche involviert. Güterabwägungen betreffen nicht nur den Bereich des medizinischen Wissens, sondern auch die Seite des Patienten, der Patientin. Dabei ist auch zu berücksichtigen, dass letztere sich - psychologisch betrachtet - in einer Abhängigkeitssituation befinden. Berücksichtigen von "Autonomie - Übelvermeidung - Fürsorge und Gerechtigkeit» [2] gilt heute sowohl im angelsächsischen wie im europäischen Kulturraum als anerkanntes Prinzip medizinisch-ethischen Handelns.

Solchen Betrachtungsweisen parallel geht die zunehmende Tendenz, alles möglichst objektivieren und verrechtlichen $\mathrm{zu}$ wollen.

Zum "Objektivieren" möchte ich kommentieren, dass Versachlichen doch auch meint, man habe alles im Griff, es gebe keine blinden Flecke oder «black boxes», dass naturwissenschaftlich alles fassbar sei und Komplexität klar in ihre einzelnen Bestandteile zerlegt werden könne.

Weiter unten soll ausgeführt werden, dass durch die Unterscheidung zwischen «Körper» und «Leib» das Imponderable, das Geheimnisvolle und Wunderbare menschlichen Lebens und seiner Entwicklung in utero hochgehalten werden soll. Meines Erachtens wäre es eine gefährliche Versuchung, an vollständige Objektivierbarkeit zu glauben. Gerade in der pluralistischen Gesellschaft mit Zerfall tradierter und/oder religiöser Werte sollte sich unser Berufsstand der Öffentlichkeit gegenüber klar für das Anerkennen von Lebensgeheimnissen, die aller naturwissenschaftlichen Erkenntnis und aller technischen Fortschritte zum Trotz weiterbestehen, einsetzen. Es sei hier nur noch das Stichwort der Totipotenz embryonaler Stammzellen erwähnt!

0. Guillod [3] sagt vom Recht, es mache sich zur Aufgabe, endgültig zwischen konfliktuellen Interessen zu entscheiden. Es ist für alle verbindlich. Im Bereich der ärztlichen Tätigkeit sind es die Bundesgesetze über die Freizügigkeit des Medizinalpersonals, über die Krankenversicherung, das Obligationenrecht und die kantonalen Gesundheitsgesetze (sowie zahlreiche Rechtsurteile). Das Recht gründet auf ein freies, verantwortliches und mit intrinsischer Würde ausgestattetes Menschenbild sowie auf Werten (etwa den Grundsätzen von Gerechtigkeit, Loyalität und Nichtschädigung), deren Schutz und Förderung es garantiert. Zum Schluss seiner Ausführungen betont Guillod [3], dass «die Regulierung der medizinischen Praktiken uns an die Grenzen von Recht, Deontologie und Ethik führen». Voraussetzung zu einer Verständigung sei, «dass jeder versucht, die Sprache des anderen, seine Denkweise, zu verstehen in einem Wort, sich der Interdisziplinarität zu stellen».

Der Begriff des "informed consent" bewegt sich meines Erachtens gerade in diesem interdisziplinären Raum und muss weiter unten - wenn die Sicht der ungewollt schwangeren Frau beleuchtet wird genauerer Betrachtung unterzogen werden. Hier sei nur soviel gesagt:

Gerade dieser Begriff des «informierten Zustimmens" bedingt den Dialog zwischen Hilfesuchendem und Helfer. Dieses Zwischenmenschliche wiederum führt weg von der reinen Objektivierung des Menschlichen hin zu einer Wiederherstellung der Person als Subjekt, so dass die Medizin wirklich relationellen Charakter erhält und behält [4]. Ethik des Arztes und 
Ethik des Patienten stehen in einer solchen Betrachtungsweise gleichwertig nebeneinander.

\section{Verantwortung als ethischer Grundbegriff}

In unserer Zivilisation hat - in Zusammenhang mit dem Prinzip der Autonomie - das «Prinzip Verantwortung" eine wachsende moralische Bedeutung bekommen. Spaemann führt zum Begriff der Verantwortung aus:

"Von Verantwortung ist nicht dort die Rede, wo genaue Handlungsanweisungen zu befolgen sind, sondern da, wo jemand für die Ordnung eines bestimmten komplexen Lebensbereiches oder für die Erledigung einer komplexen Aufgabe zuständig ist, wo er zur Wahrnehmung dieser Aufgabe einen Ermessensspielraum in eigener Kompetenz auszufüllen hat und wo er schliesslich für das Resultat seiner Handlungen rechenschaftspflichtig ist. In komplexen Situationen muss der Mensch sich flexibel erweisen, umdisponieren können; aber: umdisponieren kann nur, wer Kompetenz besitzt. Dazu kommt, dass immer mehr Menschen mehrere Rollen zu übernehmen haben.» [5]

Rollen schreiben bestimmte Verhaltensweisen vor. Koordination verschiedener Rollen erfordert Entscheidungen ausserhalb von vorprogrammiertem Verhalten.

Die moderne Technik hat zwar menschliches Handeln vielerorts durchschaubarer und ausserordentlich effizient gemacht. Zugleich führt die Wissensvermehrung aber $\mathrm{zu}$ mehr Verantwortlichkeiten. Und weil Lebensverhältnisse dazu tendieren, sich sehr rasch zu ändern, wird es je länger desto schwieriger, sich überhaupt auf ein gewisses festes Handlungsrepertoire abstützen zu können. Sowohl auf seiten der Ärzteschaft wie auch auf seiten der betroffenen Frauen gilt es, sich des oben genannten Ermessensspielraums und der daran gebundenen Rechenschaftspflicht bewusst zu sein.

Ethische Prinzipien (Autonomie, Fürsorge, Gerechtigkeit) werden heute ausserdem gemeinsam mit tugendethischen Ansätzen diskutiert. Dazu sei kurz ausgeführt: Verantwortung - so Spaemann [5] "ergibt sich stets aus Situationen, in denen wir uns befinden, aus sittlichen Verhältnissen. Sittliche Verhältnisse sind: Freundschaft, Ehe, das Verhältnis zwischen Eltern und Kindern, zwischen Arzt und Patient, zwischen Lehrern und Schülern, zwischen Berufskollegen. Sittliche Verantwortung aber ist die adäquate, sachgerechte, nicht durch Egoismus, Leidenschaft oder Fanatismus verzerrte Realisierung sittlicher Verhältnisse.»

Wenn wir den Versuch wagen, obige Aussagen auf unser Thema der Fristenregelung anzuwenden, erkennen wir unschwer, dass wir es mit komplexen Verhältnissen zu tun haben, dass individuelle Momente eine grosse Rolle spielen, dass sowohl Arzt oder Ärztin als auch die betroffenen Frauen sich dem Anspruch verschiedener Rollen zu stellen haben.

\section{Güterabwägungen}

Für den Arzt gilt es in Zusammenhang mit unserer Fragestellung zu berücksichtigen, dass hinsichtlich der Gesundheitsgefährdung durch eine Schwangerschaft nicht nur eine körperliche, sondern auch eine psychische oder soziale Schädigung der betroffenen Frau erfasst werden muss. Schwanger sein bedeutet nicht nur, einem Embryo seine somatische Entwicklung zu ermöglichen, sondern zu begreifen, dass es dabei - abgesehen von der biologischen Lebensentwicklung - auch um das Bereitstellen möglichst günstiger Voraussetzungen $\mathrm{zu}$ einer gesunden psychischen und sozialen Entwicklung eines werdenden Menschen geht. Wenn sich die Schwangere dazu nicht in der Lage fühlt, sollte der Arzt dies aufzunehmen imstande sein.

Bezieht er allerdings den gezeugten Embryo mit ein, wird die Situation sehr komplex, nicht zuletzt weil dem Embryo nur ein moralisches, keine reales Interesse [5] zukommt.

Von ärztlicher Seite wird nicht selten angeführt, dass ungewollt schwangere Frauen unter dem Druck von aussenstehenden Dritten stünden und somit gar nicht ihre autonomen Entscheidungsschritte zur Geltung brächten. Es ist dann Aufgabe des konsultierten Arztes, diese Sachlage mit der Betroffenen zu klären; denkbar - und in der Realität sicher belegbar - ist dann, dass es zum Bejahen der eingetretenen Schwangerschaft kommen kann, dass also der betroffenen Frau durch eine Bedenkfrist möglich wird, ihren wirklich autonomen Entscheid heranreifen zu lassen. Genau gleich möglich bleibt, dass die Bürde der ungewollten Schwangerschaft zu schwer bleibt, so dass die Betroffene den Abbruch wirklich von sich aus wünscht.

Der Ethiker Sass [6] sieht im Bereich der Medizinethik eine sich abzeichnende «kopernikanische Wende»: die klassischen Interventionsmaximen des hippokratischen "nil nocere» und des «bonum facere» veränderten sich durch die Eigenverantwortlichkeit auf seiten der Patienten. Die ethische Urteils- und Entscheidungsgewalt liege nicht mehr schwergewichtig beim Arzt, sondern partnerschaftlich beim Arzt und Patienten. Seitens des Arztes müssen folgende Überlegungen miteinbezogen werden: Erkennen individueller oder gruppenspezifischer Risikofaktoren, Entwickeln von Strategien zur Verringerung oder Vermeidung individueller Gesundheitsrisiken und des Erreichens von individuellen und gesellschaftlichen Gesundheitszielen.

Angesichts einer unerwünschten/ungewollten Schwangerschaft sehen Ärztin oder Arzt sich zwei Situationen gegenüber: einerseits geht es um das Verstehen der psychodynamischen Problematik, also Einfühlen und kritisches Betrachten der emotionalen, psychosozialen Überforderungen, Engpässe und Sackgassen, von denen die Betroffene spricht, um dann abzuwägen, ob aufgrund der erkannten psychischen Struktur und den gegebenen persönlichen psychischen Ressourcen das Austragen einer Schwangerschaft zumutbar sein könnte oder nicht. Dann aber 
geht es andererseits auch noch um die praktische Durchführung eines Schwangerschaftsabbruchs. Nach dem heute geltenden Gesetz sind diese beiden Komponenten recht klar voneinander getrennt, braucht es doch das Einverständnis von zwei Kolleginnen oder Kollegen, meist eines Gynäkologen oder des Hausarztes auf der einen, einer Psychiaterin oder eines Psychiaters auf der andern Seite. Für einmal dürfte die Aufgabe im Bereich Psychiatrie in vielen Fällen leichter fallen als im chirurgisch-operativen, weil ersterer nicht mit dem direkten Anblick des abgetriebenen Embryo konfrontiert ist. Zumindest wenn seitens der Ärztin/des Arztes die eigene innere Resonanz nicht unterdrückt wird, kann es wohl für den Operateur zu mehr Gewissenskonflikten kommen als beim Psychiater. Dafür sprechen gelegentliche mündliche Mitteilungen.

Judith Pòk weist in ihrem Artikel darauf hin, dass für sie als Gynäkologin Embryo und Mutter in ihrer biologischen Existenz nicht voneinander getrennt werden können. Diese Betrachtungsweise erscheint mir nicht nur somatisch-physiologisch bedenkenswert, sondern sie beleuchtet einen ganz anderen juristisch und ethisch relevanten Gedankengang: gemäss der europäischen «Übereinkunft über Menschenrechte und Biomedizin" soll allein der Entscheid einer betroffenen Person von Gewicht sein, es dürften keinerlei Interessen von Dritten miteinbezogen werden. (Diese Argumentation galt es beispielsweise auch zu berücksichtigen, als - in Zusammenhang mit Vorarbeiten zu einer gesetzlichen Regelung von Sterilisationen - vom absolut persönlichen Recht einer urteilsunfähigen Person, eine Schwangerschaft auszutragen, die Rede war: weder die Interessen des Ungeborenen noch diejenigen der potentiellen Grosseltern durften dabei in Erwägung gezogen werden!) Stellen wir dieser Argumentation die von mir «holistisch" genannte Betrachtungsweise der unteilbaren Leiblichkeit gegenüber, geht daraus deutlich hervor, dass im Stadium der Frühschwangerschaft noch kein gesonderter rechtlicher Schutzanspruch für den Embryo abgeleitet werden müsste: es geht nicht um die Frage, ab welchem Zeitpunkt dem Embryo ein Rechtsstatus zukomme, der gleichzeitig Schutzbedürftigkeit bedeutet, sondern um die Frage, ab welchem Zeitpunkt der Embryo zum unabhängig von seiner Mutter lebensfähigen Fötus - selber ein Individuum - geworden ist. Dieser gedankliche Ansatz rückt in meinen Augen auch den Respekt vor der Person der ungewollt Schwangeren und deren eigenen auch ethischen - Reflexionen zum Pro und/oder Contra der aktuellen Konfliktsituation in den Vordergrund. Dadurch - so will mir scheinen - wird dieser konflikthafte Tatbestand besser gewürdigt: Es geht dann nicht um die Frage, ab wann einem Embryo - auch naturwissenschaftlich betrachtet - der Status "Mensch" zugeordnet werden soll/muss, sondern um die einzigartige "holistische Einheit" beginnenden Lebens in utero.
Der Ärzteschaft unterstellen zu wollen, wir würden - sollte die Fristenregelung in Kraft treten können - Abtreibungen bis kurz vor der Geburt Vorschub leisten, ist verletzend, wird uns doch damit alle Ehrfurcht vor menschlichem Leben abgesprochen.

\section{Körper, den ich habe - Leib, der ich bin}

Auch in der Schweiz ist heute weithin anerkannt, dass der Wunsch nach einem Kind, der persönlichen Entscheidungsfreiheit gemäss, in die Lebensplanung einbezogen werden kann/darf. Das Erleben von Zeugung, Schwangerschaft und Geburt hat für die Frauen gerade in seiner Konflikthaftigkeit und Ambivalenz vielfach auch zu einem bewussteren und intensiveren Einlassen auf das Geschehen geführt.

Für die unerwünscht schwangere Frau bedeutet das Feststellen, dass sie geschwängert wurde, in den allermeisten Fällen ein einschneidendes Ereignis mit schwerwiegenden Folgen. Sie erlebt an sich selber mit unausweichlicher Absolutheit den Konflikt zwischen «dem Körper, den ich habe» und «dem Leib, der ich bin" [7]. Ihr ist etwas widerfahren, das ihre Vernunft nicht gewollt hätte, das aber zu einer leibhaftigen Realität geworden ist (Spaemann weist an mehreren Stellen auf das "genitum non factum" des christlichen Credo hin [5]). Gerade die Leibhaftigkeit des Novums Frühschwangerschaft führt wohl - wenn wir den Konflikt der betroffenen Frau genau untersuchen zu dem enormen Dilemma und, je nach Situation, zu ihren Entscheidungsschwierigkeiten. Scham, Ängste, Fragen nach eigenem Versagen, Schuldgefühle, Selbstunsicherheit und weitere emotionale Faktoren können einem Offenlegen der Konflikthaftigkeit der Situation hinderlich sein, die kritisch-distanzierte Betrachtung durch die Betroffene verunmöglichen. Allerdings ist auch ein völliges Verdrängen dieser "ans Lebendige rührenden" Betroffenheit nicht auszuschliessen. Es kommt dann zum Abspalten des Leibmotivs zugunsten einer reduktionistischen Betrachtung des Körpers. All diese Faktoren gilt es einzubeziehen, wenn «informed consent" zur Diskussion steht. (Warum wohl heisst übrigens die Hebamme im Französischen "sage-femme»? Um welche Weisheit kann/konnte es da gehen?!)

Wenn es "gut geht", wird die Schwangere rechtzeitig eine ärztliche Beratung aufsuchen, andernfalls könnte der Zeitpunkt, bis zu welchem ein Abbruch erlaubt wäre, verpasst werden: solche Überlegungen haben dazu geführt, dass unter den Politikerinnen und Politikern das Beratungsobligatorium doch über längere Zeit und vertieft diskutiert wurde; der Akzent liegt dabei wohl beim Aspekt der Pflicht des Staates, menschliches Leben zu schützen. Wenn wir uns auch hier der "holistischen" Betrachtungsweise annähern, müsste/dürfte wiederum gesagt werden, dass sich die Schutzpflicht somit auf einen Leib bezöge, nämlich den mütterlichen, in welchem der frühe Embryo als Anteil inbegriffen ist. 


\section{Ethik des Bürgers und Ethik des Arztes}

Die Begriffe von Leib, Leibhaftigkeit und Leiblichkeit ermöglichen uns, von der Soma-Psyche-Spaltung Abstand zu nehmen. Ich bin versucht, den Begriff "Arzt" danebenzustellen, dem auch eine andere Gewichtung und Tonalität als dem "Mediziner» zukommen: in meinen Augen sieht der Arzt sein Gegenüber als Ganzes, während der Mediziner vorwiegend die vorliegende Pathologie betrachtet. Ein dritter, wesensverwandter Begriff in diesem Zusammenhang wäre «das Heil».

Könnte sich hier ein «dritter Weg" des Betrachtens unseres schwierigen Themas abzeichnen? Liesse sich der Zwiespalt zwischen absolutem Schutz menschlichen Lebens, also Gebot zum Austragen der Schwangerschaft, und scheinbar emotionslosem und letztlich unmoralischem und illegalem Abbruch so auflösen? Eine Möglichkeit ärztlicher Ethik, die dem durch Glaubens- oder Gewissenskonflikte geplagten Arzt und der Bürgerin sowie dem Bürger nicht minder eine erweiterte Sicht auf die Konfliktträchtigkeit der Fristenregelung eröffnen könnte?

Ausgerechnet zum Zeitpunkt der Niederschrift dieses Artikels lese ich in der NZZ vom 3./4. November 2001, dass in Portugal ein Strafprozess - in einer Tennishalle, weil kein ausreichend grosser Gerichtssaal zur Verfügung steht - gegen insgesamt 43 Frauen und Männer (Hauptangeklagte: eine Hebamme, die eine illegale "Klinik» für Abtreibungen betrieben habe) angelaufen ist. 17 Frauen sollen den Abbruch ihrer Schwangerschaft gewollt haben, ein Arzt, ein Taxifahrer sowie Pflege-, Apotheken- und Sozialpersonal stehen als Mithelfer vor Gericht. Sollten wir in unserem Land als Ärztinnen und Ärzte potentiell weiterhin solche Prozesse für möglich zulassen? Sollen Frauen in einer Notlage weiterhin als letztlich Kriminelle eingestuft werden dürfen?

Der Philosoph Gadamer gibt uns zu bedenken, dass «alle Lebensformen und Lebensgebiete rationalisieren zu wollen" einer Entwicklung gleichkomme, "die mehr und mehr die panikhafte Form einer Flucht vor der Freiheit annimmt" [8]. Es kann denn auch nicht erstaunen, dass er - an anderer Stelle - von "freigebender Fürsorge» des Arztes spricht [8]: Zum einen weist Gadamer darauf hin, dass "Gesundheit nicht etwas ist, das sich als solches bei der Untersuchung zeigt, sondern etwas, das gerade dadurch ist, dass es sich entzieht. Gesundheit ist uns also nicht ständig bewusst und begleitet uns nicht besorgt wie die Krankheit. Es ist nicht etwas, das uns zur ständigen Selbstbehandlung einlädt oder mahnt. Sie gehört zu dem Wunder der Selbstvergessenheit.» Er spricht über "die Verborgenheit der Gesundheit" und dass "Gesundheit sich in ihren eigenen Massen selbst erhält, [...] dass sie sich Standardwerte, die man auf Grund von Durchschnittserfahrungen an den Einzelfall heranträgt, als etwas Ungemässes nicht aufzwingen lässt.» [8] Und er erklärt zum Begriff des Masses: «Wenn man Gesundheit in Wahrheit nicht messen kann, so eben deswegen, weil sie ein Zustand der inneren Angemessenheit und der Übereinstimmung mit sich selber ist, die man nicht durch eine andere Kontrolle überbieten kann. Deshalb bleibt die Frage an den Patienten sinnvoll, ob er sich krank fühlt.» Dieses "innerlich Angemessene» - bei den Griechen als "Harmonie, als die masshafte Angemessenheit" verstanden - müsste die Ärztin/der Arzt eben neben allem Messbaren und Objektivierbaren im Auge behalten, wenn ein Patient nicht nur behandelt, sondern geheilt - oder eben eine ungewollt schwangere Frau beraten - werden soll.

Keine Ärztin und kein Arzt will sich letztlich der Not eines anderen Menschen verschliessen. Wir sind gleichzeitig als Bürgerinnen und Bürger aufgerufen, unsere Stimme in die Urne zu legen. Zu Recht besteht auch grösster Respekt davor, "sein zu wollen wie Gott" oder des Herrschertums über Leben und Tod bezichtigt zu werden. Der "freigebenden Fürsorge» eingedenk dürften wir aber abwägen zwischen der Sicht des Arztes und den Bürgerpflichten; vor der inneren Auseinandersetzung zwischen Objektivierung und Subjektivität/Individualität nicht auszuweichen, signalisiert gegen aussen auch, dass ich als Ärztin eine mögliche Enthumanisierung des heutigen Menschenbildes nicht einfach als unausweichliche Gegebenheit anerkenne. Gleichzeitig bin ich bereit, die Argumente der Fristenregelungsgegner gelten zu lassen, gerade weil ich nicht der Generalisierung und Objektivierung alles Menschlichen das Wort reden will. 0. Guillod schliesst seinen Artikel «Recht, Ethik und Medizin: Harmonie oder Dissonanzen?» mit folgenden Worten: «Heute sind Konvergenzen - wenn nicht gar Vermengungen - von Recht und Ethik zu beobachten. Es stellt sich die Frage, ob der Bereich der Biomedizin nicht das Experimentierfeld zur Erarbeitung neuer (juristischer, parajuristischer oder metajuristischer?) Normen ist, die dazu bestimmt sind, die ständig im Fluss befindlichen Praktiken zu begleiten.

Rahmenbedingungen zu setzen, das scheint mir die Rolle des Rechts zu sein. Das bedeutet zum einen, Grenzen zu setzen (so wie der Rahmen ein Gemälde begrenzt), die von den Grundwerten zwingend gegeben (und von Ethik wie Recht anerkannt) sind - etwa Schutz des Lebens und der menschlichen Gesundheit sowie Förderung der Autonomie. Doch Grenzen zu setzen, erlaubt es, vermehrte Rechtssicherheit zu garantieren und Freiräume innerhalb des Rahmens zu schaffen.

Allein den Meisterwerken gebührt ein Rahmen. Durch den Rahmen werden sie in Szene gesetzt. Idealerweise müsste das auch für die juristische Begleitung der medizinischen Praktiken der Fall sein.» [3]

\section{Zum Schluss}

"Panta rhei»: Der Entscheid an der Urne wird dem neusten Stand des Irrtums entsprechen. Wichtig ist, dass wir uns den gesellschaftlichen Veränderungen nicht verschliessen. Gleichzeitig gilt dem «Wunder des Lebens" als zentralem Anliegen unserer Berufung weiterhin unser grosser Respekt. 


\section{Literatur}

1 Bondolfi A. Neuere Tendenzen und grundsätzliche Überlegungen zum Verhältnis von Medizin und Ethik. In: Bondolfi A, Müller H (Hrsg.). Medizinische Ethik im Alltag. Basel: EMH Schweizerischer Ärzteverlag AG; 1999. S. 17-38.

2 Bondolfi A. Die moralischen Prinzipien ethischen Handelns. In: Bondolfi A, Müller H (Hrsg.). Medizinische Ethik im Alltag. Basel: EMH Schweizerischer Ärzteverlag AG; 1999. S. $83-122$

3 Guillod 0. Recht, Ethik und Medizin: Harmonie oder Dissonanzen? In: Bondolfi A, Müller H (Hrsg.). Medizinische Ethik im Alltag. Basel: EMH Schweizerischer Ärzteverlag AG; 1999. S. 63-82
4 Kress JJ. Die Sicht eines Psychiaters. In: Tröhler U, Reiter-Theil S. Ethik und Medizin 1947-1997. Was leistet die Kodifizierung von Ethik? Göttingen: Wallstein Verlag; 1997. S. 513-5.

5 Spaemann R. Grenzen. Zur ethischen Dimension des Handelns. Stuttgart: Klett-Cotta; 2001.

6 Sass H-M. Medizinethik. In: Pieper A, Thurnherr U. Angewandte Ethik. Beck'sche Reihe BsR 1261. München: Verlag C. H. Beck; 1998. S. 80-109.

7 Graf Dürckheim K. Vom doppelten Ursprung des Menschen. Herderbücherei Band 911, 7. Auflage. Freiburg i.Br.: Verlag Herder; 1983.

8 Gadamer HG. Über die Verborgenheit der Gesundheit. Frankfurt a.M.: Suhrkamp Verlag; 1993. 